\title{
Evaluation and 3D-Fem Analysis for Contact Strength of Ceramic Plate in Contact with a Round Bar*
}

\author{
${\text { Yutaka } \mathrm{ABE}^{* *} \text {, Manabu TAKAHASHI }}^{* * *}$, Xia $\mathrm{ZHU}^{* * *}$ and Nagatoshi OKABE ${ }^{* * *}$ \\ ${ }^{* *}$ Graduate student, Graduate school of Science and Engineering, Ehime University, \\ 3 Bunkyo-cho, Matsuyama, Ehime, 790-8577 Japan \\ E-mail: yutaka.abe@toshiba.co.jp \\ ***Graduate school of Science and Engineering, Ehime University, \\ 3 Bunkyo-cho, Matsuyama, Ehime, 790-8577 Japan
}

\begin{abstract}
Stress distributions beneath the contact surface of ceramic plat in contact with round bar were analyzed by 3-D FEM. On the basis of analytical results, the contact strength was evaluated by considering varied sizes and positions of pre-existing defects from a viewpoint of fracture mechanics. The contact rupture occurs from a surface defect that pre-exists in the vicinity of the edge of the plate. If we posit the premise that a defect size and a distance from contact boundary to its defect, the contact rapture load can be evaluated by using the proposed equation.
\end{abstract}

Key words: Ceramics, Contact Fracture, Hertz Stress, Stress Gradient, Fracture Toughness

\section{Introduction}

Ceramic materials have already been applied to various structural elements such as automobile, bearing of precision rotating machine and so on [1-3]. The expansion to the engineering applications also caused various contact problems. Time dependence of strength of ceramic materials is evaluated by tensile/bending strength, effective volume and effective loading time, which is unified strength estimation method [4] that is proposed by Okabe et al. This method is applied to uniform or shelving stress distribution. Unfortunately, it cannot sufficiently evaluate the strength in steep stress distribution caused in the contact and joint conditions. It is extremely important for strength design to consider these steep stress distributions because the failure of ceramics is caused only by insensible growth of a pre-defect. As part of the study of the chipping damage and compression failure, authors reported on the contact problems of a sphere/sphere [5] and a plate/sphere $[6,7]$ of brittle materials. These papers are reported the usefulness for the strength design evaluating from a viewpoint of fracture mechanics.

Study on contact problem of a plate/ round bar [8] reported influences of horizontal and normal force in ideal contact state that is assumed an infinite plate and round bar. However, since actual contact is shape of limited, it is necessary to clarify the influence of the edge of contact. In the purpose of this paper, the contact stress distributions between round bar and plate of ceramics is analyzed by 3-D elasticity finite element method. Contact strength is discussed from a viewpoint of fracture mechanics based on the contact stress distribution analyzed. Furthermore, the contact fracture load and contact strength is evaluated by an equation which is considered sizes and positions of pre-existing defect. 


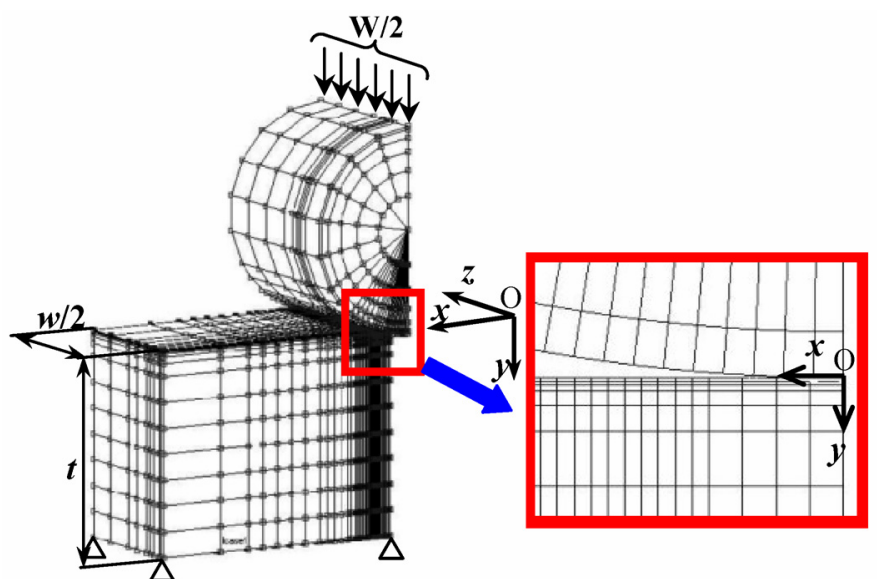

Fig. 1 Quarter model for 3-D FEM analysis in a rectangular specimen in contact with round bar.

Table 1 Mechanical properties of $\mathrm{Si}_{3} \mathrm{~N}_{4}$ used

\begin{tabular}{cccc}
\hline Young's modulus & Poisson's ratio & \multicolumn{2}{c}{ Fracture toughness } \\
$\mathrm{E}(\mathrm{GPa})$ & $v$ & $\mathrm{~K}_{\mathrm{IC}}$ & $\left(\mathrm{MPa} \cdot \mathrm{m}^{1 / 2}\right)$ \\
\hline 304 & 0.28 & & 5.0 \\
\hline
\end{tabular}

\section{FINITE ELEMENT MODELLING AND ANALYSIS METHOD}

Contact stress distributions between round bar of $3 \mathrm{~mm}$ in diameter and a rectangular plate of ${ }^{t} 3 \times{ }^{w} 4 \times{ }^{l} 10 \mathrm{~mm}$ in sintered silicon nitride were analyzed using 3-D elasticity finite element program (MSC. Marc). Analysis model was shown in Fig.1 as quarter of a rectangular specimen in contact with a round bar. This specimen was put on a metallic plate with smooth surface. The directions of $x$ and $z$ on the other side of the contact line were fixed. The length of a round bar is only $0.5 \mathrm{~mm}$ longer than the half of the width of the rectangular specimen. To consider the influence of Hertz stress generated in the contact boundary and in the plate edge $(z=0)$, the elements of those areas were subdivided. Minimum element size is about $15 \mu \mathrm{m}$. This model was meshed using 8-nodes and approximately 7500 solid elements. Mechanical properties of $\mathrm{Si}_{3} \mathrm{~N}_{4}$ used in this study are shown in Table 1. As a contact conditions, the model was given only compulsion displacement $u$ to top of the round bar. Displacement increment $\Delta u$ was gradually added separately for 15-20 steps so that the displacement rate might correspond to $0.5 \mathrm{~mm} / \mathrm{min}$. Total contact load $W(=P \cdot L)$ was obtained by summing vertical load caused in all elements of the specimen bottom. $P$ is contact load per contact length. The origin $\mathrm{O}$ of the coordinate is located in the edge of specimen on the center of contact position.

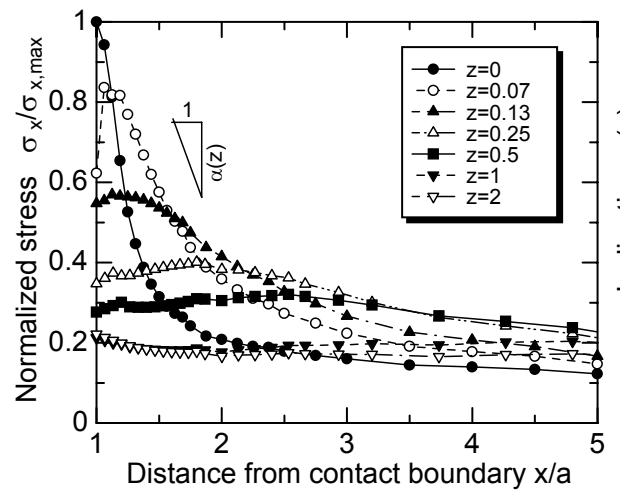

Fig. 2 Distributions of normalized stress at position $z$

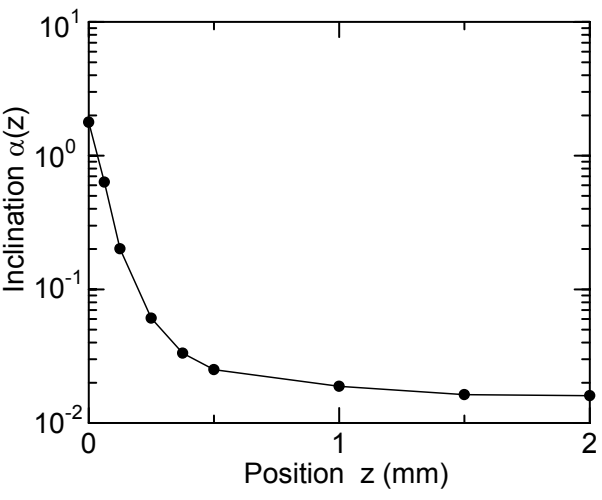

Fig. 3 Relation between position $z$ and inclinations of stress distributions $\alpha(z)$ 


\section{ANALYSIS RESULTS}

\subsection{Stress distributions beneath contact surface toward width direction}

On the basis of FEM results, maximum stress $\sigma_{x, \max }$ appeared on contact boundary $(x / a=$ $1)$ at edge of specimen $(z=0)$. Stress $\sigma_{x}$ beneath contact surface along $x / a=0$ was normalized by $\sigma_{x, \max }$. Figure 2 shows the distributions of normalized stress $\sigma_{x} / \sigma_{x, \max }$ to distance from contact boundary $x / a$ at position $z$ where is a distance from the edge of specimen width. The maximum tensile stress $\sigma_{\mathrm{x}, \max }(z)$ was moved away from contact boundary with increasing position $z$. Half length $a$ of contact width with round bar is calculated by $[9,10]$

$$
a=\sqrt{\frac{4 R W}{\pi}} \sqrt{\left(\frac{1-v_{1}^{2}}{E_{1}}+\frac{1-v_{2}^{2}}{E_{2}}\right)}
$$

where, $R$ is a sphere radius and sub-index 1 and 2 are a sphere and a rectangular specimen, respectively. In this study, $E_{1}=E_{2}$ and $v_{l}=v_{2}$.

The inclinations of stress distributions decreasing from maximum tensile stress $\sigma_{x, \max }(z)$ are defined as $\alpha(z)$. The relation between position $z$ and $\alpha(z)$ is shown in Fig. 3. The stress drastically decreases from the edge $(z=0 \mathrm{~mm})$ to about $0.3 \mathrm{~mm}$. Then, the stress inclinations slightly decrease with increasing of position $\mathrm{z}$, and level off from about $0.5 \mathrm{~mm}$.

\subsection{Stress distributions toward thickness direction}

Distributions of normalized stress $\sigma_{x} / \sigma_{x, \max }$ to a distance ( $y$-axis) from contact surface at arbitrary of $\mathrm{x} / \mathrm{a}$ are showed as Fig. 4. Stress $\sigma_{x}$ rapidly shifts from the tension to compression beneath the contact surface. On the other hand, it was confirmed that the inclinations of stress distributions show the tendency to decrease toward x-direction from the contact boundary. Therefore, tensile stress regions that involved in formation of the crack are merely depth of a few $\mu \mathrm{m}$.

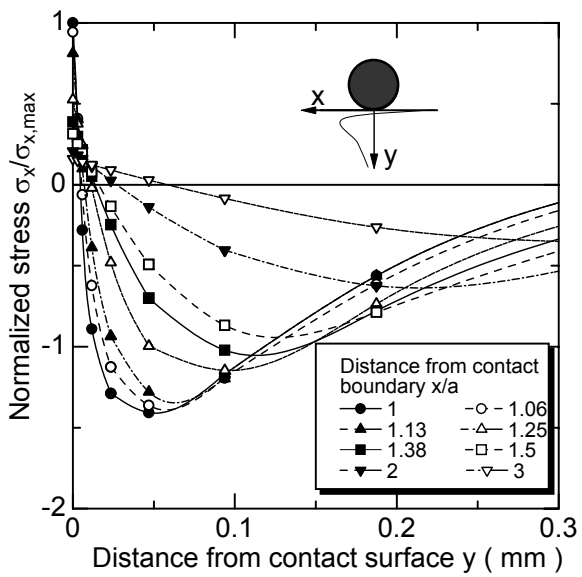

Fig. 4 Stress distributions toward thickness direction of plate

\section{DISCUSSIONS}

\subsection{Relationship between stress intensity factor and surface crack}

On the basis of observation results of the fracture surface after contact test, it was found that the fracture origin was caused from contact plate surface. Hertz stress occurs beneath the plate surface and a pre-existing surface defect influences fracture. It is assumed that the 


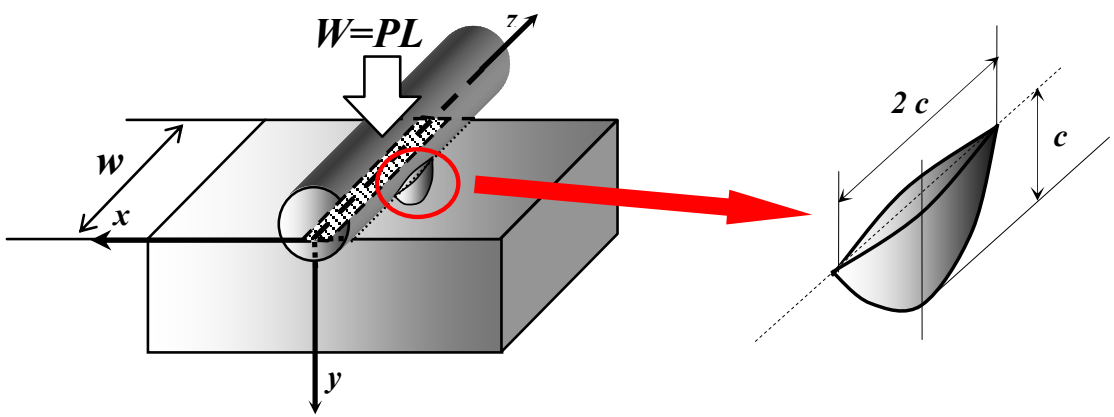

Fig. 5 Model of a three-dimensional semicircle surface crack
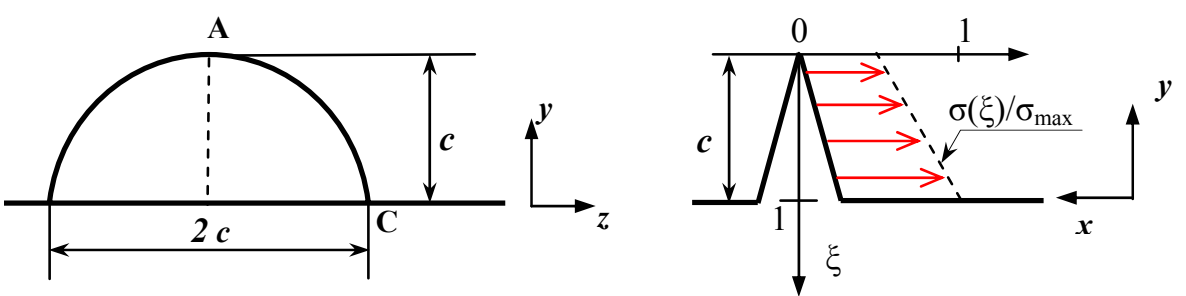

Fig. 6 Weight function for a semi-elliptical surface crack under stress distribution

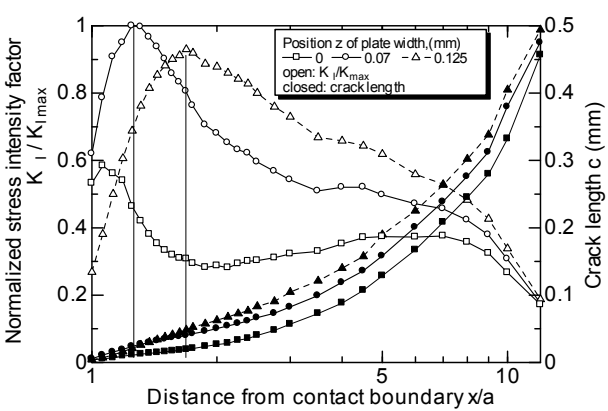

(a) in the vicinity of edge $(z=0)$

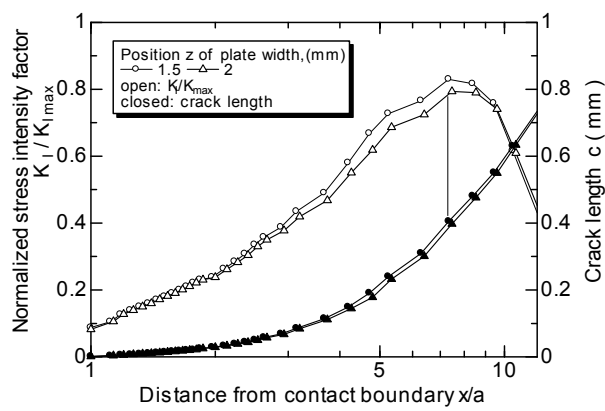

(b) in the vicinity of center $(z=2)$

Fig. 7 Relation between normalized stress intensity factor and crack length toward an arbitrary distance from the contact boundary

three-dimensional semicircle crack exists in the plate surface as shown in Fig.5. Stress intensity factor $K_{I}$ is calculated about crack length $c$ at various $z$-positions. Since tensile stress field beneath the plate surface linearly decreases toward $z$-direction, the stress distribution around a surface crack is shown as Fig.6. Stress intensity factor is calculated by the following equation [11]:

$$
\begin{aligned}
& K_{I}=n_{1} K_{1}+n_{2} K_{0}, \quad K_{i}=\frac{M_{i} \sigma_{\max } \sqrt{\pi c}}{\phi} \\
& \sigma(\xi)=\left(n_{1} \xi+n_{2}\right) \sigma_{r_{\text {max }}}(x, 0), \quad \xi=1-\frac{y}{c}
\end{aligned}
$$

where, $M$ is weight function, $\phi$ is the complete integral of the second kind and $n_{1}, n_{2}$ are coefficients in approximation of stress distribution.

Figure 7 shows the relation between normalized stress intensity factor $\mathrm{K}_{\mathrm{I}} / \mathrm{K}_{\mathrm{I} \text {,max }}$ and crack length $c$ toward an arbitrary distance $x / a$ from the contact boundary; (a) In the vicinity of edge $(z=0)$, the position of $\left(\mathrm{K}_{\mathrm{I}} / \mathrm{K}_{\mathrm{I}, \max }\right)_{\max }$ is located adjacent to the contact boundary. Critical crack length to failure is almost equal to the pre-existing defect size of usual ceramics. (b) In the vicinity of center, the positions of $\left(\mathrm{K}_{\mathrm{I}} / \mathrm{K}_{\mathrm{I}, \max }\right)_{\max }$ gradually go away from the contact boundary. Critical crack length is more than $400 \mu \mathrm{m}$. Therefore, if plate is failed by surface crack on vicinity of a half width $(z=2)$, it is an inferior material. Because the 


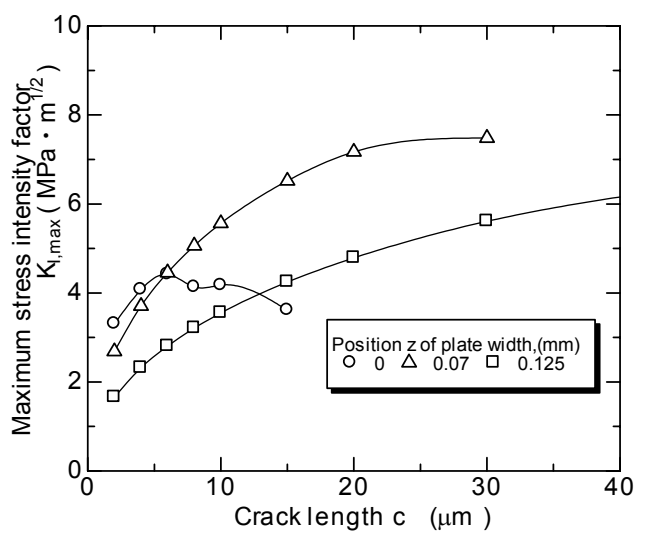

Fig. 8 Relation of maximum stress intensity factor to crack length under contact loading $(2.5 \mathrm{kN} / \mathrm{mm})$

pre-existing defects length are less than $100 \mu \mathrm{m}$ in ceramics.

Figure 8 shows the relation of maximum stress intensity factor to crack length under contact load $(2.5 \mathrm{kN} / \mathrm{mm})$. When the pre-existing defect length is assumed a range from 6 to $30 \mu \mathrm{m}, \mathrm{K}_{\mathrm{I}}$ indicates maximum value on $z=0.07$. It suggests that the edge of plate be origins of fracture.

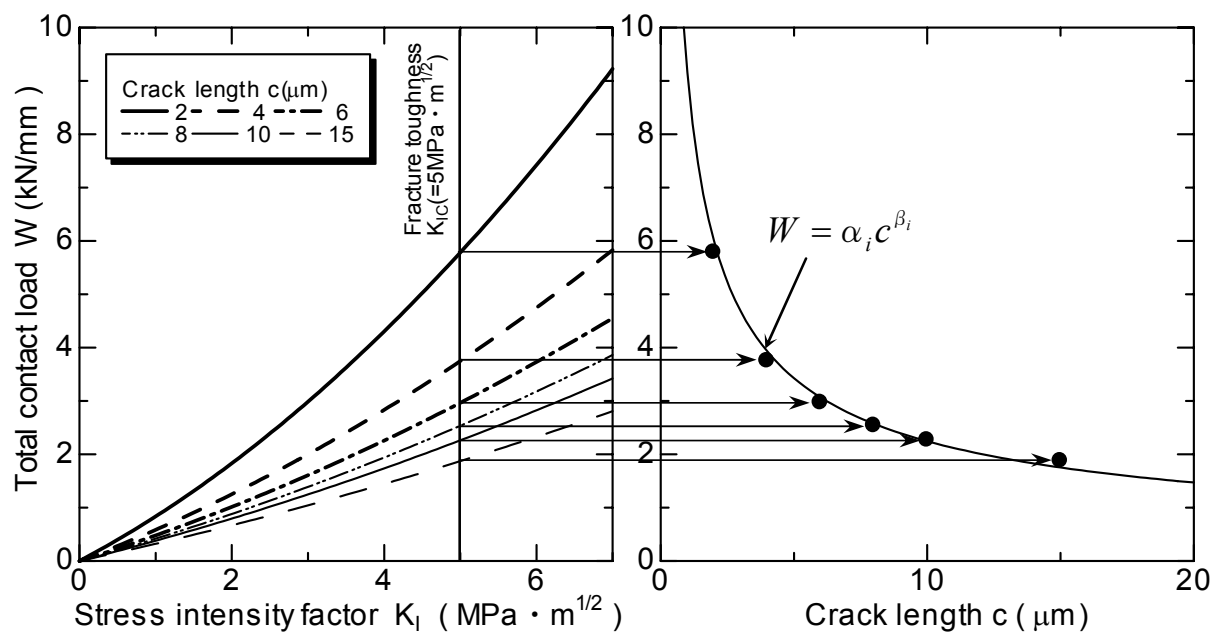

Fig. 9 Relation between stress intensity factor $\mathrm{K}_{\mathrm{I}}$ and contact load at an arbitrary distance $x_{i}(x / a=1.187)$

Fig. 10 Relation of contact load to crack length $\mathrm{c}$ at an arbitrary distance $x_{i}(x / a=1.187)$

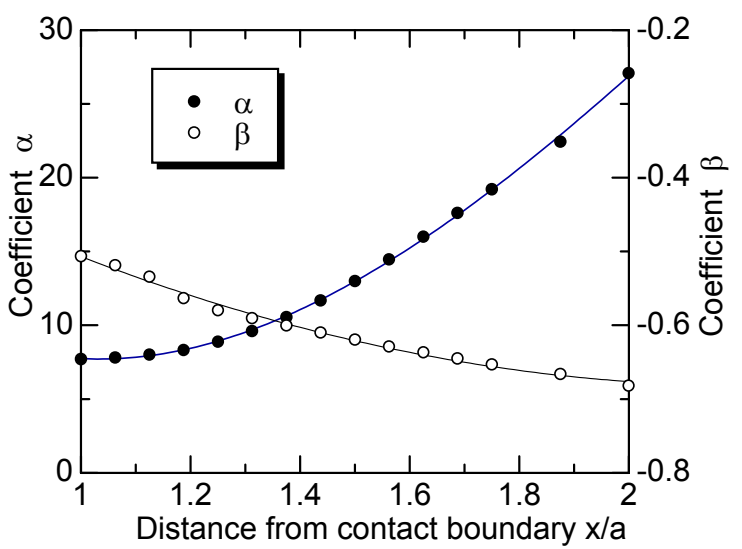

Fig. 11 Relation of coefficient $\alpha, \beta$ and position of a defect. 


\subsection{Strength evaluation including size and position of pre-existing defects}

One of the relations between stress intensity factor and total contact load at an arbitrary distance $x_{i}$ from the contact boundary is shown in Fig. 9. When the stress intensity factor in the surface crack reaches the fracture toughness value of ceramics $\left(\mathrm{K}_{\mathrm{IC}}=5 \mathrm{MPa} \cdot \mathrm{m}^{1 / 2}\right)$, it is assumed that the specimen is failed rapidly. Then, the total contact load $W$ was calculated in case a defect existed at an arbitrary distance $x_{i}$. The relation between crack length $c$ and the total contact load $W$ on distance $x_{i}$ can be shown as Fig. 10.

Using this relation, the relation between the crack length and the total contact load $W_{i}$ can be shown as following approximate equation at an arbitrary distance $x_{i}$.

$$
W_{i}=\alpha_{i} c^{\beta_{i}} \quad(i=1,2,3, \cdots n)
$$

where, $\alpha\left(c_{\mathrm{i}}\right)$ and $\beta\left(c_{\mathrm{i}}\right)$ are coefficient derived from Eq. (2).

In addition, when the coefficients of $\alpha$ and $\beta$ are arranged as a function of $x$ as shown in Fig. 11, the total contact fracture load $W_{c}$ considering a position of a defect is given by

$$
W_{c}(c, x)=\alpha^{*}(x) c^{\beta^{*}(x)}
$$

where, $\alpha^{*}$ and $\beta^{*}$ are coefficient considering a defect size and its position that involved in stress intensity factor. Therefore, if defect size $c$ and distance $x$ from contact boundary to its defect are clarified, the total contact load $W$ can evaluate by using Eq. (4).

Furthermore, it is important to evaluate the contact fracture strength. From the discussion about mentioning above, if we posit the premise that position $z=0.07$ where the stress intensity factor that indicated the maximum value is a fracture origin. Figure 12 shows

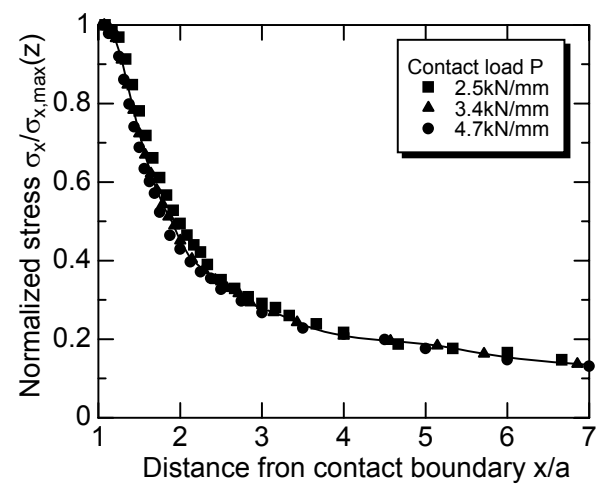

Fig. 12 Normalized stress distribution of $x$-direction to various contact loads at $z=0.07$.

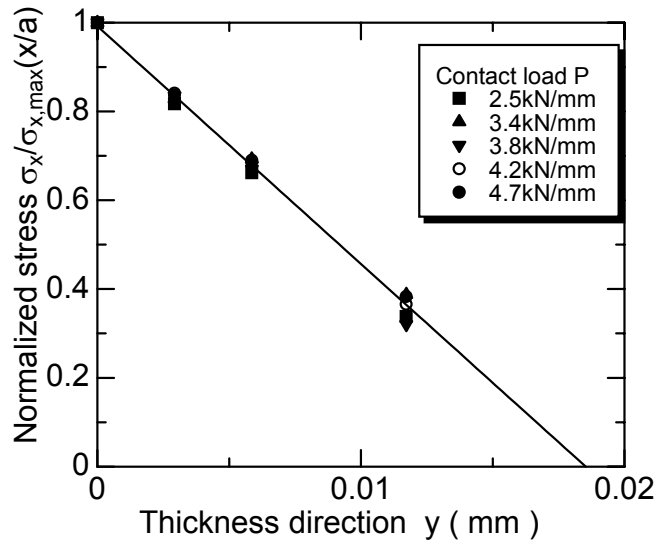

Fig. 13 Normalized stress distribution of $y$-direction to various contact load at $x / a=1.2$ and $z=0.07$. 


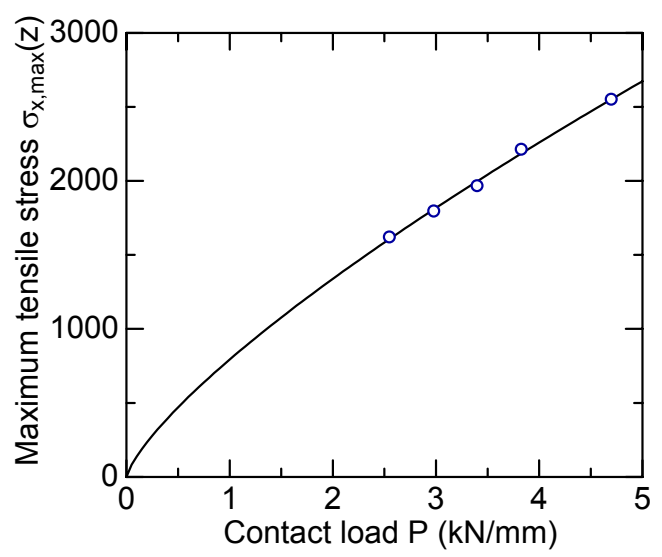

Fig. 14 Relationship between contact load and maximum tensile stress.

normalized stress distribution of $x$-direction on plate surface $(y=0)$ to various contact loads $\mathrm{P}(\mathrm{kN} / \mathrm{mm})$. The normalized stress distributions can be arranged as one curve that doesn't depend on the contact load. Figure 13 shows normalized stress distribution of $y$-direction to various contact load at $x / a=1.2$. Then, the contact stress distribution near the contact boundary can be approximated as the following equation:

$$
\frac{\sigma_{x}}{\sigma_{x, \max }}=\exp \left(-A \frac{x}{a}\right)^{B}+\exp \left\{C\left(1-\frac{x}{a}\right)\right\}^{D}
$$

where, A, B, C and D are coefficient. The relation between contact load and maximum tensile stress is shown as Fig. 14. This relation was approximated as follows:

$$
\sigma_{x, \max }=k P^{m}
$$

where, $k$ and $m$ are coefficient. Using Eqs. (5) and (6), the relation between the contact load $\mathrm{P}$ and the contact stress $\sigma_{x}$ is given by the following equation:

$$
\sigma_{x}=k P^{m} \cdot\left[\exp \left(-A \frac{x}{a}\right)^{B}+\exp \left\{C\left(1-\frac{x}{a}\right)\right\}^{D}\right]
$$

If the total contact load $W$ is already known, Hertz type stress distribution beneath the contact surface can be calculated. Moreover, if a total contact load is measured by contact test, the contact fracture strength can be evaluated. Finally, as the future work, we should clarify the relation of those coefficients and material property, and size effects etc.

\section{CONCLUSIONS}

1. Maximum tensile stress indicated beneath contact boundary $(x=a)$ at edge of specimen $(z=0)$. The position of maximum tensile stress $\sigma_{\mathrm{x}, \max }(z)$ showed a tendency away toward $z$-direction.

2. Maximum stress intensity factor $\mathrm{K}_{\mathrm{I}, \max }$ is located in $1.1-1.2$ times of the contact boundary, and the crack length involved in failure is short because stress distribution is steep. The position of $\mathrm{K}_{\mathrm{I} \text {,max }}$ gradually goes away from the contact boundary toward width direction, therefore, the crack length involved in failure is long.

3. If defect size and distance from contact boundary to its defect can assume, the contact stress $\sigma_{x}$ can evaluate by using the evaluation equation. 


\section{References}

(1) Tsuchiya, T., Development of Ceramic Gas Turbine for Integrated Coal Gasification Combined Cycle" , Journal of Gas Turbine Society, Japan, 18-69 (1990), pp.6-9. in Japanese

(2) Hara, Y. et al. "Development of a 20MW Class Ceramic Gas Turbine for Power Generation - Progress on Ceramic Component Development “, Proc. '91, Yokohama, Int. Gas Turbine Congr., IGTC, (1991)

(3) Ikeda,T., et al., Study of the Ceramic Application for Heavy Duty Gas-Turbine Blades, ASME paper, 91-GT, (1991), p.372.

(4) Okabe, N. and Hirata, H., High temperature fatigue properties for some types of SiC and Si3N4 and the unified strength estimation method, Cyclic fatigue in ceramics, C.J.M.R-14, The Society of Material Science, Japan, (1995), pp.245-276.

(5) Abe,Y., et al., Sampling inspection method for ring crack initiation strength of ceramic rolling bearing balls, Transactions of the Japan Society of Mechanical Engineers, Series A, Vol.61, No.589 (1995), pp.1884-1889.

(6) Takahashi, M., Okabe, N. and Izumi, N., Contact strength and probabilistic estimation of glass, The Society of Material Science, Japan, 53-2 (2004), pp.175-181. in Japanese

(7) Takahashi, M., Okabe, N., Zhu, X. and Matsui, M., Initiation strength of ring-crack caused by sphere-plate contact of ceramics, The Society of Material Science, Japan, 53-10(2004), pp.1149-1155. in Japanese

(8) Abe, Y., Okabe, N. and Takahashi, M., Influences of horizontal force and round-bar contact on contact strength of ceramics, Transactions of the Japan Society of Mechanical Engineers, Series A, in press.

(9) Timoshenko S., Theory of Elasticity, (1934), p.339.

(10) Smith J.O., Journal of applied Mechanics, (1953), p.157.

(11) Eds. Murakami Y., et al. Stress intensity factors handbook, Pergamon Press, 2, (1987), p.723. 